\title{
DISTILLED SENSING: ADAPTIVE SAMPLING FOR SPARSE DETECTION AND ESTIMATION
}

\author{
By Jarvis Haupt*, Rui Castro ${ }^{\dagger}$, And Robert NowaK \\ Rice University*, Columbia University ${ }^{\dagger}$, and \\ University of Wisconsin-Madison ${ }^{\ddagger}$
}

\begin{abstract}
Adaptive sampling results in dramatic improvements in the recovery of sparse signals in white Gaussian noise. A sequential adaptive sampling-and-refinement procedure called distilled sensing (DS) is proposed and analyzed. DS is a form of multi-stage experimental design and testing. Because of the adaptive nature of the data collection, DS can detect and localize far weaker signals than possible from non-adaptive measurements. In particular, reliable detection and localization (support estimation) using non-adaptive samples is possible only if the signal amplitudes grow logarithmically with the problem dimension. Here it is shown that using adaptive sampling, reliable detection is possible provided the amplitude exceeds a constant, and localization is possible when the amplitude exceeds any arbitrarily slowly growing function of the dimension.
\end{abstract}

1. Introduction. In high dimensional multiple hypothesis testing problems the aim is to identify the subset of the hypotheses that differ from the null distribution, or simply to decide if one or more of the hypotheses do not follow the null. There is now a well developed theory and methodology for this problem, and the fundamental limitations in the high dimensional setting are quite clear. However, most existing treatments of the problem assume a non-adaptive measurement process. The question of how the limitations might differ under a more flexible, sequential adaptive measurement process has not been addressed. This paper shows that this additional flexibility can yield surprising and dramatic performance gains.

For concreteness let $x=\left(x_{1}, \ldots, x_{p}\right) \in \mathbb{R}^{p}$ be an unknown sparse vector, such that most (or all) of its components $x_{i}$ are equal to zero. The locations of the non-zero components are arbitrary. This vector is observed in additive

\footnotetext{
$\S$ This work was supported in part by NSF Grant CCF-0353079 and AFOSR Grant FA9550-09-1-0140, and is dedicated to the memory of Dr. Dennis Healy, who inspired and supported this direction of research in the context of the DARPA Analog-to-Information Program. Dennis' guidance, vision, and inspiration will be missed.

AMS 2000 subject classifications: Primary 62L05, 62G20; secondary 62H15, 62P10

Keywords and phrases: Adaptive sampling, model selection, multiple testing, sequential design, sparse recovery
} 
white Gaussian noise and we consider two problems:

Localization: Infer the locations of the few non-zero components.

Detection: Decide whether $x$ is the zero vector.

Given a single, non-adaptive noisy measurement of $x$, a common approach entails coordinate-wise thresholding of the observed data at a given level, identifying the number and locations of entries for which the corresponding observation exceeds the threshold. In such settings there are sharp asymptotic thresholds that the magnitude of the non-zero components must exceed in order for the signal to be localizable and/or detectable. Such characterizations have been given in $[1,7]$ for the localization problem and $[11,6]$ for the detection problem. A more thorough review of these sort of characterizations is given in Section 2.1.

In this paper we investigate these problems under a more flexible measurement process. Suppose we are able to sequentially collect multiple noisy measurements of each component of $x$, and that the data so obtained can be modeled as

$$
y_{i, j}=x_{i}+\gamma_{i, j}^{-1 / 2} w_{i, j}, i=1, \ldots, p, \quad j=1, \ldots, k .
$$

In the above a total of $k$ measurement steps is taken, $j$ indexes the measurement step, $w_{i, j} \stackrel{\text { i.i.d. }}{\sim} \mathcal{N}(0,1)$ are zero-mean Gaussian random variables with unit variance, and $\gamma_{i, j} \geq 0$ quantifies the precision of each measurement. When $\gamma_{i, j}=0$ we adopt the convention that component $x_{i}$ was not observed at step $j$. The crucial feature of this model is that it does not preclude sequentially adaptive measurements, where the $\gamma_{i, j}$ can depend on past observations $\left\{y_{i, \ell}\right\}_{i \in\{1, \ldots, p\}, \ell<j}$.

In practice, the precision for a measurement at location $i$ at step $j$ may be controlled, for example, by collecting multiple independent samples and averaging to reduce the effective observation noise, the result of which would be an observation described by the model (1.1). In this case, the parameters $\left\{\gamma_{i, j}\right\}$ can be thought of as proportional to the number of samples collected at location $i$ at step $j$. The precision parameters might also, in some settings, be interpreted as time parameters, where $\left\{\gamma_{i, j}\right\}$ would be proportional to the length of time for which the component at location $i$ is observed at step $j$.

In order to make fair comparisons to non-adaptive measurement processes, the total precision budget is limited in the following way. Let $R(p)$ be an increasing function of $p$, the dimension of the problem (that is, the number of hypotheses under scrutiny). The precision parameters $\left\{\gamma_{i, j}\right\}$ are required 
to satisfy

$$
\sum_{j=1}^{k} \sum_{i=1}^{p} \gamma_{i, j} \leq R(p) .
$$

For example, the usual non-adaptive, single measurement model corresponds to taking $R(p)=p, k=1$, and $\gamma_{i, 1}=1$ for $i=1, \ldots, p$. This baseline can be compared with adaptive procedures by keeping $R(p)=p$, but allowing $k>1$ and variables $\left\{\gamma_{i, j}\right\}$ satisfying (1.2).

The multiple measurement process (1.1) is applicable in many interesting and relevant scenarios. For example in gene association and expression studies, two-stage approaches are gaining popularity (see [13, 17, 14] and references therein): in the first stage a large number of genes is initially tested to identify a promising subset of them, and in the second-stage these promising genes are subject to further testing. Such ideas have been extended to multiple-stage approaches; see, for example [18]. More broadly, sequential experimental design has been popular in other fields as well, such as in computer vision where it is known as active vision [16], or in machine learning, where it is known as active learning [4, 5]. These types of procedures can potentially impact other areas such as microarray-based studies and astronomical surveying.

There are two main contributions of this paper. First, we propose a sequential, adaptive measurement algorithm called distilled sensing (DS). The idea behind DS is simple: use a portion of the precision budget to crudely measure all components; eliminate a fraction of the components that appear least promising from further consideration after this measurement; and iterate this procedure several times, at each step measuring only components retained after the previous step. While similar procedures have been proposed in experimental science $[17,18,14]$, to the best of our knowledge the quantification of performance gains has not been addressed. Our second main contribution is a theoretical analysis that reveals the dramatic gains that can be attained by the use of such sequential procedures. The preliminary version of this work appeared at the 12th International Conference on Artificial Intelligence and Statistics (AISTATS) [10]; here we significantly extend the results for the localization problem and provide a novel characterization of the detection problem.

This paper is organized as follows. Following a brief discussion of the fundamental limits of non-adaptive sampling for detection and localization in Sect. 2.1, our main result - that DS can reliably solve the localization and detection problems for dramatically weaker signals than what is possible using non-adaptive measurements - is stated in Sect. 2.2. A proof of the main 
result is given in Sect. 3. Simulation results demonstrating the theory are provided in Sect. 4, and conclusions and extensions are discussed in Sect. 5. A proof of the threshold for localization from non-adaptive measurements and several auxiliary lemmata are provided in the appendices.

2. Main Results. The main results of our theoretical analysis of distilled sensing (DS) are stated in this section, but first we begin by reviewing the asymptotic thresholds for localization and detection from non-adaptive measurements. As mentioned above, these thresholds are now well known $[1,7,11,6]$, but here we provide a concise summary of the main ideas, in terms that will facilitate our comparison with DS. We then highlight some of the surprising gains achievable through adaptive measurement using DS.

2.1. Non-adaptive Localization and Detection of Sparse Signals. The nonadaptive measurement model we will consider as the baseline for comparison is as follows. We have a single observation of $x$ in noise:

$$
y_{i}=x_{i}+w_{i}, i=1, \ldots, p
$$

where $w_{i} \stackrel{\text { i.i.d. }}{\sim} \mathcal{N}(0,1)$. As noted above, this is a special case of our general setup (1.1) in which $k=1$ and $\gamma_{i, 1}=1$ for $i=1, \ldots, p$. This implies a precision budget $R(p)=\sum_{i=1}^{p} \gamma_{i, 1}=p$.

To describe the asymptotic (large $p$ ) thresholds for localization we need to introduce some notation. Define the false-discovery proportion (FDP) and non-discovery proportion (NDP) as follows.

DeFinition 2.1. Let $\mathcal{S}:=\left\{i: x_{i} \neq 0\right\}$ denote the signal support set and let $\widehat{\mathcal{S}}=\widehat{\mathcal{S}}(y)$ denote an estimator of $\mathcal{S}$. The false-discovery proportion is

$$
\operatorname{FDP}(\widehat{\mathcal{S}}):=\frac{|\widehat{\mathcal{S}} \backslash \mathcal{S}|}{|\widehat{\mathcal{S}}|} .
$$

In words, the FDP of $\widehat{\mathcal{S}}$ is the ratio of the number of components falsely declared as non-zero to the total number of components declared non-zero. The non-discovery proportion is

$$
\operatorname{NDP}(\widehat{\mathcal{S}}):=\frac{|S \backslash \widehat{\mathcal{S}}|}{|S|}
$$

In words, the $\mathrm{NDP}$ of $\widehat{\mathcal{S}}$ is the ratio of the number of non-zero components missed to the number of actual non-zero components. 
In this paper we focus in particular on the scenario where $x_{i} \geq 0$ for all $i \in\{1, \ldots, p\}$. We elaborate on possible extensions in Section 2.2. Under this assumption it is quite natural to focus on a specific class of estimators of $\mathcal{S}$.

Definition 2.2. A coordinate-wise thresholding procedure is an estimator of the following form:

$$
\widehat{\mathcal{S}}_{\tau}(y):=\left\{i \in\{1, \ldots, p\}: y_{i} \geq \tau>0\right\},
$$

where the threshold $\tau$ may depend implicitly on $x$, or on $y$ itself.

The following result establishes the limits of localization using non-adaptive sampling. A proof is provided in Appendix A; see also [8], where related results were obtained under a random signal model.

Theorem 2.3. Assume $x$ has $p^{1-\beta}, \beta \in(0,1)$, non-zero components of amplitude $\sqrt{2 r \log p}, r>0$, and measurement model (2.1). There exists a coordinate-wise thresholding procedure that yields an estimator $\widehat{\mathcal{S}}=\widehat{\mathcal{S}}(y)$ such that if $r>\beta$, then as $p \rightarrow \infty$,

$$
\operatorname{FDP}(\widehat{\mathcal{S}}) \stackrel{P}{\rightarrow} 0, \quad \operatorname{NDP}(\widehat{\mathcal{S}}) \stackrel{P}{\rightarrow} 0
$$

where $\stackrel{P}{\rightarrow}$ denotes convergence in probability. Moreover, if $r<\beta$, then there does not exist a coordinate-wise thresholding procedure that can guarantee that both quantities above tend to 0 as $p \rightarrow \infty$.

The detection problem, which amounts to a hypothesis test between the null distribution $x=0$ and a sparse alternative, has also been addressed in the literature under a random signal model $[11,6]$. Consider the hypothesis testing problem:

$$
\begin{aligned}
& \mathrm{H}_{0} \quad: \quad y_{i} \stackrel{\mathrm{iid}}{\sim} \mathcal{N}(0,1), i=1, \ldots, p \\
& \mathrm{H}_{1}: y_{i} \stackrel{\mathrm{iid}}{\sim}(1-\theta(p)) \mathcal{N}(0,1)+\theta(p) \mathcal{N}(\mu(p), 1), i=1, \ldots, p
\end{aligned}
$$

These hypotheses model measurements of either the zero vector, or of a randomly generated signal $x$, with each entry having amplitude $\mu(p)=\sqrt{2 r \log p}$ independently with probability $\theta(p)=p^{-\beta}$, and amplitude zero with probability $1-p^{-\beta}$, according to the measurement model (2.1). Note that under the alternative, the signal has $p^{1-\beta}$ non-zero components in expectation. We recall the following result. 
Theorem 2.4. Consider the hypotheses in (2.2) where $\mu(p)=\sqrt{2 r \log p}$. Define

$$
\rho(\beta):= \begin{cases}0, & 0<\beta \leq 1 / 2 \\ \beta-1 / 2, & 1 / 2<\beta \leq 3 / 4 \\ (1-\sqrt{1-\beta})^{2}, & 3 / 4<\beta<1\end{cases}
$$

If $r>\rho(\beta)$, then there exists a test for which the sum of the Type 1 and Type 2 errors tends to 0 as $p \rightarrow \infty$. Conversely, if $r<\rho(\beta)$, then for any test the sum of Type I and Type II errors tends to 1 as $p \rightarrow \infty$.

Theorem 2.4 was proved in [6] relying heavily on the ideas presented in [11]. Although it is stated for a random sparsity model, it is possible to relate the results to the deterministic sparsity model that we consider in the paper, namely using the ideas presented in Chapter 8 of [12].

2.2. Distilled Sensing: Adaptive Localization and Detection of Sparse Signals. Algorithm 1 describes the DS measurement process. At each step of the process, we retain only the components with non-negative observations. This means that when the number of non-zero components is very small, roughly half of the components are eliminated from further consideration at each step. Consequently, if the precision budget allocated at each step is slightly larger than $1 / 2$ of that used in the preceding step, then the effective precision of the measurements made at each step is increasing. In particular, if the budget for each step is $1 / 2+c$ of the budget at the previous step, for some small constant $c>0$, then the precision of the measured components is increasing exponentially. Therefore, the key is to show that the very crude thresholding at 0 at each step does not remove a significant number of the non-zero components. One final observation is that because the number of components measured decreases by a factor of roughly $1 / 2$ at each step, the total number of measurements made by DS is roughly $2 p$, a modest increase relative to the $p$ measurements made in the non-adaptive setting.

Recall from above that for non-adaptive sampling, reliable detection and localization is only possible provided the signal amplitude is $\Omega(\sqrt{\log (p)})$. In other words, the signal amplitude must exceed a constant (that depends on the sparsity level) times $\sqrt{\log (p)}$. The following theorem establishes that DS is capable of detecting and localizing much weaker sparse signals. For the purposes of our investigation we assume that the non-zero components are positive. It is trivial to extend the algorithm and its analysis to handle both positive and negative components by simply repeating the entire process 


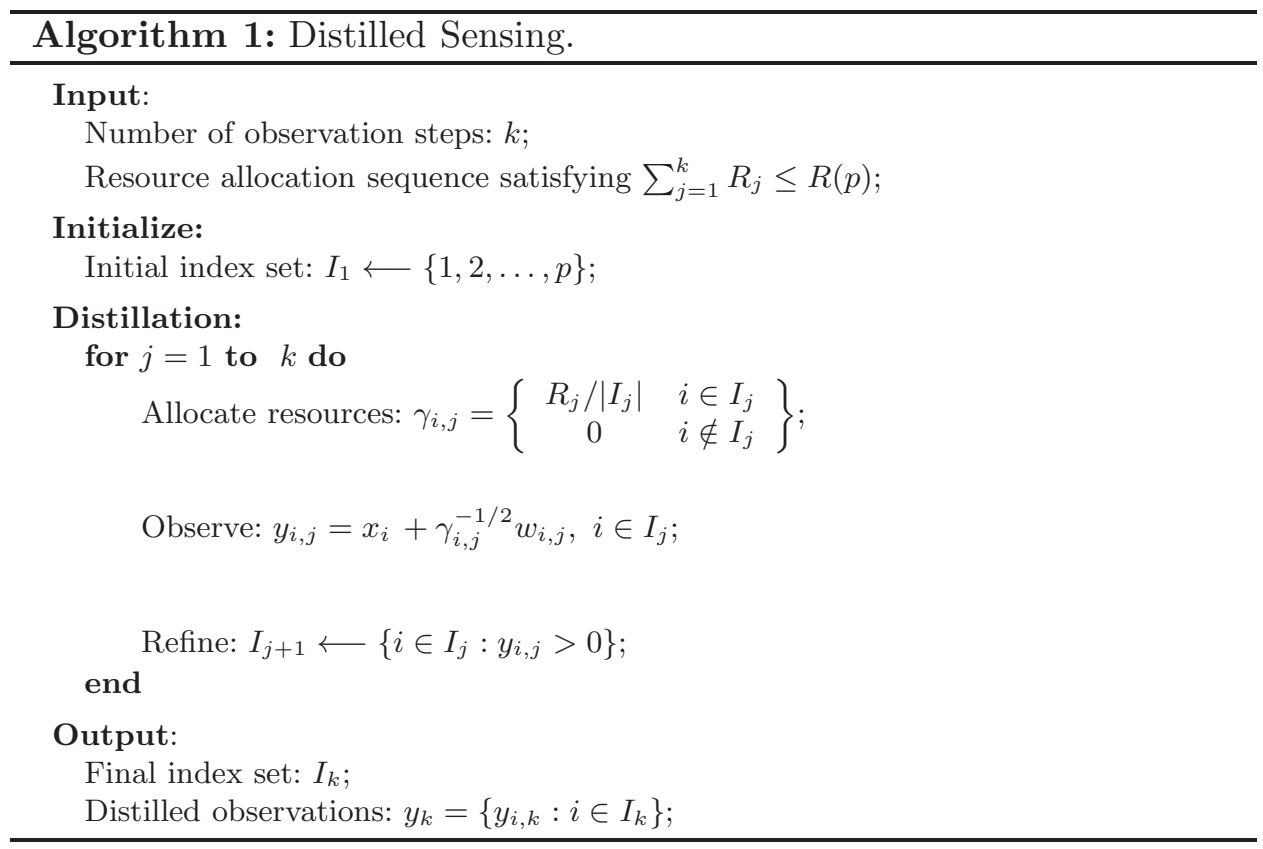

twice; once as described, and again with $y_{i, j}$ replaced with $-y_{i, j}$ in the refinement step of Algorithm 1.

Theorem 2.5. Assume $x \geq 0$ with $p^{1-\beta}, \beta \in(0,1)$, non-zero components of amplitude $\mu(p)$, and sequential measurement model using Distilled Sensing with $k=k_{p}=\max \left\{\left\lceil\log _{2} \log p\right\rceil, 0\right\}+2$, and precision budget distributed over the measurement steps so that $\sum_{j=1}^{k} R_{j} \leq p, R_{j+1} / R_{j} \geq \delta>$ $1 / 2$, and $R_{1}=c_{1} p$ and $R_{k}=c_{k} p$ for some $c_{1}, c_{k} \in(0,1)$. Then the support set estimator constructed using the output of the DS algorithm

$$
\widehat{\mathcal{S}}_{\mathrm{DS}}:=\left\{i \in I_{k}: y_{i, k}>\sqrt{2 / c_{k}}\right\}
$$

has the following properties:

(i) if $\mu(p)$ is any positive diverging sequence in $p$, then as $p \rightarrow \infty$

$$
\operatorname{FDP}\left(\widehat{\mathcal{S}}_{\mathrm{DS}}\right) \stackrel{P}{\rightarrow} 0, \quad \operatorname{NDP}\left(\widehat{\mathcal{S}}_{\mathrm{DS}}\right) \stackrel{P}{\rightarrow} 0 .
$$

(ii) if $\mu(p)>\max \left\{\sqrt{4 / c_{1}}, 2 \sqrt{2 / c_{k}}\right\}$ (a constant) then

$$
\lim _{p \rightarrow \infty} \operatorname{Pr}\left(\widehat{\mathcal{S}}_{\mathrm{DS}}=\emptyset\right)=\left\{\begin{array}{ll}
1, & \text { if } x=0 \\
0, & \text { if } x \neq 0
\end{array},\right.
$$

where $\emptyset$ is the empty set. 
In words, this result states that DS successfully identifies the sparse signal support provided only that the signal amplitude grows (arbitrarily slowly) as a function of the problem dimension $p$, while reliable signal detection requires only that the signal amplitude exceed a constant. Comparison with the $\Omega(\sqrt{\log p})$ amplitude required for both tasks using non-adaptive sampling illustrates the dramatic gains that are achieved through adaptivity.

3. Analysis of Distilled Sensing. In this section we prove the main result characterizing the performance of distilled sensing (DS), Theorem 2.5. We begin with three lemmas that quantify the finite sample behavior of DS.

\subsection{Distillation: Reject the Nulls, Retain the Signal.}

LEMmA 3.1. If $\left\{y_{i}\right\}_{i=1}^{m} \stackrel{\text { iid }}{\sim} \mathcal{N}\left(0, \sigma^{2}\right), \sigma>0$, then for any $0<\varepsilon<1 / 2$,

$$
\left(\frac{1}{2}-\varepsilon\right) m \leq\left|\left\{i \in\{1, \ldots, m\}: y_{i}>0\right\}\right| \leq\left(\frac{1}{2}+\varepsilon\right) m,
$$

with probability at least $1-2 \exp \left(-2 m \varepsilon^{2}\right)$.

Proof. For any event $A$, let $\mathbf{1}_{A}$ be the indicator taking the value 1 if $A$ is true and 0 otherwise. By Hoeffding's inequality, for any $\varepsilon>0$

$$
\operatorname{Pr}\left(\left|\sum_{i=1}^{m} \mathbf{1}_{\left\{y_{i}>0\right\}}-\frac{m}{2}\right|>m \varepsilon\right) \leq 2 \exp \left(-2 m \varepsilon^{2}\right) .
$$

Imposing the restriction $\varepsilon<1 / 2$ guarantees that the corresponding fractions are bounded away from zero and one.

Lemma 3.2. Let $\left\{y_{i}\right\}_{i=1}^{m} \stackrel{\mathrm{iid}}{\sim} \mathcal{N}\left(\mu, \sigma^{2}\right)$, with $\sigma>0$ and $\mu \geq 2 \sigma$. Define $\epsilon=\frac{\sigma}{\mu \sqrt{2 \pi}}<1$. Then

$$
(1-\epsilon) m \leq\left|\left\{i \in\{1,2, \ldots, m\}: y_{i}>0\right\}\right| \leq m,
$$

with probability at least $1-\exp \left(-\frac{\mu m}{4 \sigma \sqrt{2 \pi}}\right)$.

Proof. We will utilize the following standard bound on the Gaussian tail: for $Z \sim \mathcal{N}(0,1)$ and $\gamma>0$,

$$
\frac{1}{\sqrt{2 \pi \gamma^{2}}}\left(1-\frac{1}{\gamma^{2}}\right) \exp \left(-\gamma^{2} / 2\right) \leq \operatorname{Pr}(Z>\gamma) \leq \frac{1}{\sqrt{2 \pi \gamma^{2}}} \exp \left(-\gamma^{2} / 2\right)
$$


Let $q=\operatorname{Pr}\left(y_{i}>0\right)$, then it follows that

$$
1-q \leq \frac{\sigma}{\mu \sqrt{2 \pi}} \exp \left(-\frac{\mu^{2}}{2 \sigma^{2}}\right)
$$

Next we use the Binomial tail bound from [3]: for any $0<b<\mathbb{E}\left[\sum_{i=1}^{m} \mathbf{1}_{\left\{y_{i}>0\right\}}\right]=$ $m p$,

$$
\operatorname{Pr}\left(\sum_{i=1}^{m} \mathbf{1}_{\left\{y_{i}>0\right\}} \leq b\right) \leq\left(\frac{m-m p}{m-b}\right)^{m-b}\left(\frac{m p}{b}\right)^{b} .
$$

Note that $\epsilon>1-q$ (or equivalently, $1-\epsilon<q$ ), so we can apply this result to $\sum_{i=1}^{m} \mathbf{1}_{\left\{y_{i}>0\right\}}$ with $b=(1-\epsilon) m$ to obtain

$$
\begin{aligned}
\operatorname{Pr}\left(\sum_{i=1}^{m} \mathbf{1}_{\left\{y_{i}>0\right\}} \leq(1-\epsilon) m\right) & \leq\left(\frac{1-q}{\epsilon}\right)^{\epsilon m}\left(\frac{q}{1-\epsilon}\right)^{(1-\epsilon) m} \\
& \leq \exp \left(-\frac{\mu^{2} \epsilon m}{2 \sigma^{2}}\right)\left(\frac{1}{1-\epsilon}\right)^{(1-\epsilon) m} .
\end{aligned}
$$

Now, to establish the stated result, it suffices to show

$$
-\frac{\mu^{2}}{2 \sigma^{2}}+\left(\frac{1-\epsilon}{\epsilon}\right) \log \left(\frac{1}{1-\epsilon}\right) \leq-\frac{\mu}{4 \epsilon \sigma \sqrt{2 \pi}}=-\frac{\mu^{2}}{4 \sigma^{2}}
$$

which holds provided $\mu \geq 2 \sigma$, since $0<\epsilon<1$ and $\left(\frac{1-\epsilon}{\epsilon}\right) \log \left(\frac{1}{1-\epsilon}\right) \leq 1$ for $\epsilon \in(0,1)$.

3.2. The Output of the DS Procedure. Refer to Algorithm 1 and define $s_{j}:=\left|\mathcal{S} \cap I_{j}\right|$ and $z_{j}:=\left|\mathcal{S}^{c} \bigcap I_{j}\right|$, the number of non-zero and zero components, respectively, present at the beginning of step $j$, for $j=1, \ldots, k$. Let $\varepsilon>0$, and for $j=1, \ldots, k-1$ define

$$
\epsilon_{j}^{2}:=\frac{s_{1}+(1 / 2+\varepsilon)^{j-1} z_{1}}{2 \pi \mu^{2} R_{j}}
$$

The output of the DS procedure is quantified in the following result.

LEMmA 3.3. Let $0<\varepsilon<1 / 2$ and assume that $R_{j}>\frac{4}{\mu^{2}}\left(s_{1}+(1 / 2+\varepsilon)^{j-1} z_{1}\right)$, $j=1, \ldots, k-1$. If $|\mathcal{S}|>0$, then with probability at least

$$
1-\sum_{j=1}^{k-1} \exp \left(\frac{-s_{1} \prod_{\ell=1}^{j-1}\left(1-\epsilon_{\ell}\right)}{2 \sqrt{2 \pi}}\right)-2 \sum_{j=1}^{k-1} \exp \left(-2 z_{1}(1 / 2-\varepsilon)^{j-1} \varepsilon^{2}\right)
$$


$\prod_{\ell=1}^{j-1}\left(1-\epsilon_{\ell}\right) s_{1} \leq s_{j} \leq s_{1}$ and $\left(\frac{1}{2}-\varepsilon\right)^{j-1} z_{1} \leq z_{j} \leq\left(\frac{1}{2}+\varepsilon\right)^{j-1} z_{1}$ for $j=$ $2, \ldots, k$. If $|\mathcal{S}|=0$, then with probability at least

$$
\begin{array}{r}
1-2 \sum_{j=1}^{k-1} \exp \left(-2 z_{1}(1 / 2-\varepsilon)^{j-1} \varepsilon^{2}\right), \\
\left(\frac{1}{2}-\varepsilon\right)^{j-1} z_{1} \leq z_{j} \leq\left(\frac{1}{2}+\varepsilon\right)^{j-1} z_{1} \text { for } j=2, \ldots, k .
\end{array}
$$

Proof. The results follow from Lemmas 3.1 and 3.2 and the union bound. First assume that $s_{1}=|\mathcal{S}|>0$. Let $\sigma_{j}^{2}:=\left|I_{j}\right| / R_{j}=\left(s_{j}+z_{j}\right) / R_{j}$ and $\widetilde{\epsilon}_{j}:=\frac{\sigma_{j}}{\mu \sqrt{2 \pi}}, j=1, \ldots, k$.

The argument proceeds by conditioning on the output of all prior refinement steps; in particular, suppose that $\left(1-\widetilde{\epsilon}_{\ell-1}\right) s_{\ell-1} \leq s_{\ell} \leq s_{\ell-1}$ and $\left(\frac{1}{2}-\varepsilon\right) z_{\ell-1} \leq z_{\ell} \leq\left(\frac{1}{2}+\varepsilon\right) z_{\ell-1}$ for $\ell=1, \ldots, j$. Then apply Lemma 3.1 with $m=z_{j}$, Lemma 3.2 with $m=s_{j}$ and $\sigma^{2}=\sigma_{j}^{2}$, and the union bound to obtain that with probability at least

$$
1-\exp \left(-\frac{\mu s_{j}}{4 \sigma_{j} \sqrt{2 \pi}}\right)-2 \exp \left(-2 z_{j} \varepsilon^{2}\right),
$$

$\left(1-\widetilde{\epsilon}_{j}\right) s_{j} \leq s_{j+1} \leq s_{j}$, and $\left(\frac{1}{2}-\varepsilon\right) z_{j} \leq z_{j+1} \leq\left(\frac{1}{2}+\varepsilon\right) z_{j}$. Note that the condition $R_{j}>\frac{4}{\mu^{2}}\left(s_{1}+(1 / 2+\varepsilon)^{j-1} z_{1}\right)$ and the assumptions on prior refinement steps ensure that $\mu>2 \sigma_{j}$, which is required for Lemma 3.2. The condition $\mu>2 \sigma_{j}$ also allows us to simplify probability bound (3.2), so that the event above occurs with probability at least

$$
1-\exp \left(-\frac{s_{j}}{2 \sqrt{2 \pi}}\right)-2 \exp \left(-2 z_{j} \varepsilon^{2}\right)
$$

Next, we can recursively apply the union bound and the bounds on $s_{j}$ and $z_{j}$ above to obtain for $j=1, \ldots, k-1$

$$
\epsilon_{j}=\sqrt{\frac{s_{1}+(1 / 2+\varepsilon)^{j-1} z_{1}}{2 \pi \mu^{2} R_{j}}} \geq \widetilde{\epsilon}_{j}=\frac{\sigma_{j}}{\mu \sqrt{2 \pi}},
$$

with probability at least

$$
1-\sum_{j=1}^{k-1} \exp \left(\frac{-s_{1} \prod_{\ell=1}^{j-1}\left(1-\epsilon_{\ell}\right)}{2 \sqrt{2 \pi}}\right)-\sum_{j=1}^{k-1} 2 \exp \left(-2 z_{1}(1 / 2-\varepsilon)^{j-1} \varepsilon^{2}\right) .
$$

Note that the condition $R_{j}>\frac{4}{\mu^{2}}\left(s_{1}+(1 / 2+\varepsilon)^{j-1} z_{1}\right)$ implies that $\epsilon_{j}<1$. The first result follows directly. If $s_{1}=|\mathcal{S}|=0$, then consider only $z_{j}$, $j=1, \ldots, k$. The result follows again by the union bound. Note that for this statement the condition on $R_{j}$ is not required. 
Now we examine the conditions $R_{j}>\frac{4}{\mu^{2}}\left(s_{1}+(1 / 2+\varepsilon)^{j-1} z_{1}\right), j=$ $1, \ldots, k$ more closely. Define $c:=s_{1} /\left[(1 / 2+\varepsilon)^{k-1} z_{1}\right]$, in effect condensing several problem-specific parameters $\left(s_{1}, z_{1}\right.$, and $\left.k\right)$ into a single scalar parameter. Then the conditions on $R_{j}$ are satisfied if

$$
R_{j}>\frac{4 z_{1}(1 / 2+\varepsilon)^{j-1}}{\mu^{2}}\left(c(1 / 2+\varepsilon)^{k-j}+1\right) .
$$

Since $z_{1} \leq p$, the following condition is sufficient

$$
R_{j}>\frac{4 p(1 / 2+\varepsilon)^{j-1}}{\mu^{2}}\left(c(1 / 2+\varepsilon)^{k-j}+1\right),
$$

and in particular the more stringent condition $R_{j}>\frac{4(c+1) p(1 / 2+\varepsilon)^{j-1}}{\mu^{2}}$ will suffice. It is now easy to see that if $s_{1} \ll z 1$ (e.g., so that $c \leq 1$ ), then the sufficient conditions become $R_{j}>\frac{8 p}{\mu^{2}}(1 / 2+\varepsilon)^{j-1}, j=1, \ldots, k$. Thus, for the sparse situations we consider, the precision allocated to each step must be just slightly greater than $1 / 2$ of the precision allocated in the previous step. We are now in position to prove the main theorem.

3.3. Proof of Theorem 2.5. Throughout the proof, whenever asymptotic notation or limits are used it is always under the assumption that $p \rightarrow \infty$, and we use the standard notation $f(p)=o(g(p))$ to indicate that $\lim _{p \rightarrow \infty} f(p) / g(p)=0$, for $f(p) \geq 0$ and $g(p)>0$. Also the quantities $k:=k(p), \varepsilon:=\varepsilon(p)$ and $\mu:=\mu(p)$ are functions of $p$, but we do not denote this explicitly for ease of notation. We let $\varepsilon:=p^{-1 / 3}$ throughout the proof.

We begin by proving part (ii) of the theorem, which is concerned with detecting the presence or absence of a sparse signal. Part (i), which pertains to identifying the locations of the non-zero components, then follows with a slight modification.

Case 1 - Signal absent $(\mathcal{S}=\emptyset)$ : This is the simplest scenario, but through its analysis we will develop tools that will be useful when analyzing the case where the signal is present. Here, we have $s_{1}=0$ and $z_{1}=p$, and the number of indices retained at the end of the DS procedure $\left|I_{k}\right|$ is equal to $z_{k}$. Define the event

$$
\Gamma=\left\{\left(\frac{1}{2}-\varepsilon\right)^{k-1} p \leq\left|I_{k}\right| \leq\left(\frac{1}{2}+\varepsilon\right)^{k-1} p\right\} .
$$

The second part of Lemma 3.3 characterizes the probability of this event; in particular

$$
\operatorname{Pr}(\Gamma) \geq 1-2 \sum_{j=1}^{k-1} \exp \left(-2 p\left(\frac{1}{2}-\varepsilon\right)^{j-1} \varepsilon^{2}\right)
$$


Since $k \leq \log _{2} \log p+3$, for large enough $p$ we get that

$$
\begin{aligned}
\operatorname{Pr}(\Gamma) & \geq 1-2(k-1) \exp \left(-2 p\left(\frac{1}{2}-\varepsilon\right)^{k-2} \varepsilon^{2}\right) \\
& =1-2(k-1) \exp \left(-p\left(\frac{1}{2}\right)^{k-3}(1-2 \varepsilon)^{k-2} \varepsilon^{2}\right) \\
& \geq 1-2\left(\log _{2} \log p+2\right) \exp \left(-\frac{p^{1 / 3}}{\log p}(1-o(1))\right)
\end{aligned}
$$

where we used Lemma B.1 to conclude that $(1-2 \varepsilon)^{k-2}=1-o(1)$. It is clear that $\operatorname{Pr}(\Gamma) \rightarrow 1$.

In this case we assume that $\mathcal{S}=\emptyset$, therefore the output of the DS procedure consists of $\left|I_{k}\right|$ i.i.d. Gaussian random variables with zero mean and variance $\left|I_{k}\right| / R_{k}=\left|I_{k}\right| /\left(c_{k} p\right)$. Note that given $\Gamma$,

$$
\begin{aligned}
\left|I_{k}\right| \leq p\left(\frac{1}{2}+\varepsilon\right)^{k-1} & =p \frac{1}{2}\left(\frac{1}{2}\right)^{k-2}(1+2 \varepsilon)^{k-1} \\
& \leq \frac{1}{2} \frac{p}{\log p}(1+o(1))
\end{aligned}
$$

which follows from the fact that $k \geq \log _{2} \log p+2$, and using Lemma B.1. With this in hand we conclude that (with a slight abuse of notation)

$$
\begin{aligned}
\operatorname{Pr}\left(\widehat{\mathcal{S}}_{\mathrm{DS}} \neq \emptyset \mid \Gamma\right) & =\operatorname{Pr}\left(\exists_{i \in I_{k}}: y_{i, k}>\sqrt{2 / c_{k}}\right) \\
& \leq\left|I_{k}\right| \operatorname{Pr}\left(\mathcal{N}\left(0,\left|I_{k}\right| / c_{k} p\right)>\sqrt{2 / c_{k}}\right) \\
& =\left|I_{k}\right| \operatorname{Pr}\left(\mathcal{N}(0,1)>\sqrt{2 p /\left|I_{k}\right|}\right) \\
& \leq p \operatorname{Pr}(\mathcal{N}(0,1)>\sqrt{4 \log p(1-o(1))}) \\
& \leq p \exp (-2 \log p(1-o(1))) \\
& =p^{-1+o(1) \rightarrow 0,}
\end{aligned}
$$

where the last inequality follows from the standard Gaussian tail bound. This together with $\operatorname{Pr}(\Gamma) \rightarrow 1$ immediately shows that when $\mathcal{S}=\emptyset$ we have $\operatorname{Pr}\left(\widehat{\mathcal{S}}_{\mathrm{DS}} \neq \emptyset\right) \rightarrow 0$.

Case 2 - Signal present $(\mathcal{S} \neq \emptyset)$ : The proof follows the same idea as in the previous case, although the argument is a little more involved. Begin by applying Lemma 3.3 and constructing an event that occurs with probability 
tending to one. Let $\Gamma$ be the event

$$
\begin{array}{r}
\Gamma=\left\{z_{1}\left(\frac{1}{2}-\varepsilon\right)^{k-1} \leq z_{k} \leq z_{1}\left(\frac{1}{2}+\varepsilon\right)^{k-1}\right\} \\
\bigcap\left\{s_{1} \prod_{j=1}^{k-1}\left(1-\epsilon_{j}\right) \leq s_{k} \leq s_{1}\right\},
\end{array}
$$

where $\epsilon_{j}$ is given by equation (3.1). Lemma 3.3 characterizes the probability of this event under a condition on $R_{j}$ that we will now verify. Note that this condition is equivalent to $\epsilon_{j}^{2}<1 /(8 \pi)$ for all $j=1, \ldots, k-1$. Instead of showing exactly this we will show a stronger result that will be quite useful in a later stage of the proof. Recall that $R_{j+1} / R_{j} \geq \delta>1 / 2, j=1, \ldots, k-2$, and $R_{1}=c_{1} p$ by the assumptions of the theorem. Thus for $j=1, \ldots, k-1$

$$
\begin{aligned}
\epsilon_{j}^{2} & \leq \frac{s_{1}+\left(\frac{1}{2}+\varepsilon\right)^{j-1} z_{1}}{2 \pi \mu^{2} \delta^{j-1} R_{1}} \\
& \leq \frac{1}{2 \pi \mu^{2} c_{1}}\left(\frac{s_{1}}{p} \delta^{-(j-1)}+\frac{z_{1}}{p}\left(\frac{\delta}{\frac{1}{2}+\varepsilon}\right)^{-(j-1)}\right) .
\end{aligned}
$$

Clearly we have that $\epsilon_{1}^{2} \leq \frac{1}{2 \pi \mu^{2} c_{1}}<1 /(8 \pi)$ since by assumption $\mu>\sqrt{4 / c_{1}}$. Now consider the case $j>1$. Recall that $k \leq \log _{2} \log p+3$. Therefore if $\delta \geq 1$, then the term $\delta^{-(j-1)}$ can be upper bounded by 1 , otherwise

$$
\delta^{-(j-1)} \leq \delta^{-(k-2)} \leq \delta^{-\left(\log _{2} \log p+1\right)}=\delta^{-1}(\log p)^{-\log _{2} \delta} \leq 2 \log p,
$$

where the last step follows from $\delta>1 / 2$.

Now recall that $s_{1}=p^{1-\beta}$, therefore

$$
\begin{aligned}
\epsilon_{j}^{2} & \leq \frac{1}{2 \pi \mu^{2} c_{1}}\left(p^{-\beta} \delta^{-(j-1)}+\left(\frac{\delta}{\frac{1}{2}+\varepsilon}\right)^{-(j-1)}\right) \\
& \leq \frac{1}{2 \pi \mu^{2} c_{1}}\left(2 p^{-\beta} \log p+\left(\frac{\delta}{\frac{1}{2}+\varepsilon}\right)^{-(j-1)}\right) .
\end{aligned}
$$

Note that, since $\varepsilon \rightarrow 0$ as $p \rightarrow \infty$ we have that, for $p$ large enough, $\delta /(1 / 2+$ $\varepsilon)>(\delta+1 / 2+\varepsilon)$. Assume $p$ is large enough so that this is true, then

$$
\epsilon_{j}^{2} \leq \frac{1}{2 \pi \mu^{2} c_{1}}\left(2 p^{-\beta} \log p+\left(\delta+\frac{1}{2}+\varepsilon\right)^{-(j-1)}\right) .
$$


Clearly since $j \leq k-1 \leq \log _{2} \log p+2$ we have that $\left(\delta+\frac{1}{2}+\varepsilon\right)^{-(j-1)}=$ $\Omega\left(1 /(\log p)^{\log _{2}(\delta+1 / 2+\epsilon)}\right)$ and so the first of the additive terms in (3.4) is negligible for large $p$. Therefore for $p$ sufficiently large, we have, for all $j=$ $1, \ldots, k-1$

$$
\epsilon_{j}^{2} \leq \frac{1}{2 \pi \mu^{2} c_{1}}\left(\delta+\frac{1}{2}\right)^{-(j-1)} .
$$

Since by assumption $\mu>\sqrt{4 / c_{1}}$, we conclude that, for all $p$ sufficiently large, $\epsilon_{j}^{2}<1 /(8 \pi)$ for all $j=1, \ldots, k-1$, and so $R_{j}>\frac{4}{\mu^{2}}\left(s_{1}+(1 / 2+\varepsilon)^{j-1} z_{1}\right)$ for $j=1, \ldots, k-1$. Thus, applying Lemma 3.3 we have

$$
\begin{aligned}
& \operatorname{Pr}(\Gamma) \\
& \quad \geq 1-\sum_{j=1}^{k-1} \exp \left(\frac{-s_{1} \prod_{\ell=1}^{j-1}\left(1-\epsilon_{\ell}\right)}{2 \sqrt{2 \pi}}\right)-2 \sum_{j=1}^{k-1} \exp \left(-2 z_{1}(1 / 2-\varepsilon)^{j-1} \varepsilon^{2}\right) .
\end{aligned}
$$

By a similar argument to that used in Case 1, it is straightforward to show that

$$
2 \sum_{j=1}^{k-1} \exp \left(-2 z_{1}(1 / 2-\varepsilon)^{j-1} \varepsilon^{2}\right) \rightarrow 0 .
$$

In addition,

$$
\begin{aligned}
& \sum_{j=1}^{k-1} \exp \left(\frac{-s_{1} \prod_{\ell=1}^{j-1}\left(1-\epsilon_{\ell}\right)}{\sqrt{8 \pi}}\right) \\
& \quad \leq \quad(k-1) \exp \left(\frac{-s_{1} \prod_{\ell=1}^{k-2}\left(1-\epsilon_{\ell}\right)}{\sqrt{8 \pi}}\right) \\
& \quad \leq(k-1) \exp \left(\frac{-s_{1} \prod_{\ell=1}^{k-2}\left(1-\frac{1}{\mu \sqrt{2 \pi c_{1}}}\left(\delta+\frac{1}{2}\right)^{-(\ell-1) / 2}\right)}{\sqrt{8 \pi}}\right) \\
& \quad \leq(k-1) \exp \left(\frac{-s_{1} \prod_{\ell=1}^{k-2}\left(1-\frac{1}{\sqrt{8 \pi}}\left(\delta+\frac{1}{2}\right)^{-(\ell-1) / 2}\right)}{\sqrt{8 \pi}}\right),
\end{aligned}
$$

where in the last step we used the fact that $\mu>\sqrt{4 / c_{1}}$. Finally note that from Lemma B.2 we know that

$$
\prod_{\ell=1}^{k-2}\left(1-\frac{1}{\sqrt{8 \pi}}\left(\delta+\frac{1}{2}\right)^{-(\ell-1) / 2}\right) \rightarrow L(\delta),
$$


where $L(\delta)>0$ hence

$$
\begin{aligned}
& \sum_{j=1}^{k-1} \exp \left(\frac{-s_{1} \prod_{\ell=1}^{j-1}\left(1-\epsilon_{\ell}\right)}{\sqrt{8 \pi}}\right) \\
& \quad \leq\left(\log _{2} \log p+2\right) \exp \left(\frac{-p^{1-\beta}(L(\delta)+o(1))}{\sqrt{8 \pi}}\right) \\
& \quad \rightarrow 0 .
\end{aligned}
$$

Therefore we conclude that the event $\Gamma$ happens with probability converging to one.

We now proceed as before, by conditioning on event $\Gamma$. The output of the DS procedure consists of a total of $\left|I_{k}\right|=s_{k}+z_{k}$ independent Gaussian measurements with variance $\left|I_{k}\right| / R_{k}$, where $s_{k}$ of them have mean $\mu$ and the remaining $z_{k}$ have mean zero. We will show that the proposed thresholding procedure identifies only true non-zero components (i.e., correctly rejects all the zero-valued components). In other words, with probability tending to one, $\widehat{\mathcal{S}}_{\mathrm{DS}}=\mathcal{S} \cap I_{k}$. For ease of notation, and without loss of generality, assume the $y_{i, k} \sim \mathcal{N}\left(\mu,\left|I_{k}\right| / R_{k}\right)$ for $i \in\left\{1, \ldots, s_{k}\right\}$ and $y_{i, k} \sim \mathcal{N}\left(0,\left|I_{k}\right| / R_{k}\right)$ for $i \in\left\{s_{k}+1, \ldots,\left|I_{k}\right|\right\}$. Then

$$
\begin{aligned}
\operatorname{Pr} & \left(\widehat{\mathcal{S}}_{\mathrm{DS}} \neq \mathcal{S} \cap I_{k} \mid \Gamma\right) \\
& =\operatorname{Pr}\left(\bigcup_{i=1}^{s_{k}}\left\{y_{i, k}<\sqrt{2 / c_{k}}\right\} \text { or } \bigcup_{i=s_{k}+1}^{\left|I_{k}\right|}\left\{y_{i, k}>\sqrt{2 / c_{k}}\right\} \mid \Gamma\right) \\
& \leq s_{k} \operatorname{Pr}\left(\mathcal{N}\left(\mu,\left|I_{k}\right| / R_{k}\right)<\sqrt{2 / c_{k}}\right)+z_{k} \operatorname{Pr}\left(\mathcal{N}\left(0,\left|I_{k}\right| / R_{k}\right)>\sqrt{2 / c_{k}}\right) .
\end{aligned}
$$

Note that conditioned on the event $\Gamma$ (using arguments similar to those in Case 1)

$$
\begin{aligned}
\left|I_{k}\right| & =s_{k}+z_{k} \leq s_{1}+z_{1}\left(\frac{1}{2}+\varepsilon\right)^{k-1} \\
& \leq p^{1-\beta}+\frac{p}{2 \log p}(1+o(1)) \leq \frac{p}{2 \log p}(1+o(1)) .
\end{aligned}
$$


Finally, taking into account that $\mu>2 \sqrt{2 / c_{k}}$ we conclude that

$$
\begin{aligned}
\operatorname{Pr} & \left(\widehat{\mathcal{S}}_{\mathrm{DS}} \neq \mathcal{S} \cap I_{k} \mid \Gamma\right) \\
& \leq s_{k} \operatorname{Pr}\left(\mathcal{N}\left(0,\left|I_{k}\right| / R_{k}\right)<-\sqrt{2 / c_{k}}\right)+z_{k} \operatorname{Pr}\left(\mathcal{N}\left(0,\left|I_{k}\right| / R_{k}\right)>\sqrt{2 / c_{k}}\right) \\
& \leq s_{k} \operatorname{Pr}\left(\mathcal{N}(0,1)>\sqrt{\frac{2 p}{\left|I_{k}\right|}}\right)+z_{k} \operatorname{Pr}\left(\mathcal{N}(0,1)>\sqrt{\frac{2 p}{\left|I_{k}\right|}}\right) \\
& =\left|I_{k}\right| \operatorname{Pr}\left(\mathcal{N}(0,1)>\sqrt{\frac{2 p}{\left|I_{k}\right|}}\right) \\
& \leq p \operatorname{Pr}(\mathcal{N}(0,1)>\sqrt{4 \log p(1-o(1))}) \\
& \leq p \exp (-2 \log p(1-o(1))) \\
& =p^{-1+o(1)} \rightarrow 0,
\end{aligned}
$$

where the last inequality follows from the standard Gaussian tail bound. This together with $\operatorname{Pr}(\Gamma) \rightarrow 1$, and the fact that $\left|\mathcal{S} \cap I_{k}\right|=s_{k}=L(\delta)(1-$ $o(1)) s_{1}$ is bounded away from zero for large enough $p$ immediately shows that $\operatorname{Pr}\left(\widehat{\mathcal{S}}_{\mathrm{DS}}=\emptyset\right) \rightarrow 0$, concluding the proof of part (ii) of the theorem.

Part (i) of the theorem follows from the result proved above, since if $\mu$ is any positive diverging sequence in $p$ then a stronger version of Lemma B.2 applies. In particular, recall (3.5), and note that Lemma B.2 implies

$$
\prod_{\ell=1}^{k-1}\left(1-\epsilon_{\ell}\right) \geq \prod_{\ell=1}^{k-1}\left(1-\frac{1}{\mu \sqrt{2 \pi c_{1}}}\left(\delta+\frac{1}{2}\right)^{-(\ell-1) / 2}\right) \rightarrow 1 .
$$

We have already established that the events $\Gamma$ and $\left\{\widehat{\mathcal{S}}_{\mathrm{DS}} \neq \mathcal{S} \cap I_{k}\right\}$ both hold (simultaneously) with probability tending to one. Conditionally on these events we have

$$
\operatorname{FDP}\left(\widehat{\mathcal{S}}_{\mathrm{DS}}\right)=\frac{0}{s_{k}}=0
$$

and

$$
\operatorname{NDP}\left(\widehat{\mathcal{S}}_{\mathrm{DS}}\right)=\frac{s_{1}-s_{k}}{s_{1}}=1-\frac{s_{k}}{s_{1}} \rightarrow 0
$$

since from the definition of $\Gamma$ we have

$$
s_{1} \geq s_{k} \geq s_{1} \prod_{\ell=1}^{k-1}\left(1-\epsilon_{\ell}\right) \rightarrow s_{1} .
$$

Therefore we conclude that both $\operatorname{FDP}\left(\widehat{\mathcal{S}}_{\mathrm{DS}}\right)$ and $\operatorname{NDP}\left(\widehat{\mathcal{S}}_{\mathrm{DS}}\right)$ converge in probability to zero as $p \rightarrow \infty$, concluding the proof of the theorem. 
4. Numerical Experiments. This section presents numerical experiments with distilled sensing (DS). The results demonstrate that the asymptotic analysis predicts the performance in finite dimensional cases quite well. Furthermore, the experiments suggest useful rules of thumb for implementing DS in practice.

First of all, note that in theory DS reduces the signal-to-noise ratio (SNR) required for reliable recovery by a factor of roughly $\log p$. Our experiments confirm this. With the non-adaptive sensing, an SNR of $\Omega(\log p)$ is required for the reliable detection and recovery of the sparse support set. The DS procedure provides useful improvements over the non-adaptive performance for SNRs close to 1, as the theory predicts.

There are two input parameters to the DS procedure; the number of distillation steps, $k$, and the distribution of precision across the steps, $\left\{R_{j}\right\}_{j=1}^{k}$. Throughout our simulations we use the choice $k=\max \left\{\left\lceil\log _{2} \log p\right\rceil, 0\right\}+2$, as in Theorem 2.5. For the precision distribution, first recall the discussion following the proof of Lemma 3.3. There it is argued that if the sparsity model is valid, a sufficient condition for the precision distribution is $R_{j}>R_{1}(1 / 2+\varepsilon)^{j-1}, j=1, \ldots, k$, with $0<\varepsilon<1 / 2$. In words, the precision allocated to each step must be greater than $1 / 2$ the precision allocated in the previous step. In practice, we find that choosing $R_{j+1} / R_{j}=0.75$ for $j=1, \ldots, k-2$ provides good performance over the full SNR range of interest. Also, from the proof of the main result (Theorem 2.5) we see that the threshold for detection is inversely proportional to the square root of the precision allocation in the first and last steps. Thus, we have found that allocating equal precision in the first and last steps is beneficial. The intuition is that the first step is the most crucial in controlling the NDP and the final step is most crucial in controlling the FDP. Thus, the precision allocation used throughout the simulations follows this simple formula:

$$
\begin{aligned}
& R_{j}=(0.75)^{j-1} R_{1}, j=2, \ldots, k-1, \\
& R_{k}=R_{1}
\end{aligned}
$$

and $R_{1}$ is chosen so that $\sum_{j=1}^{k} R_{j}=p$.

Figure 1 compares the FDP vs. NDP performance of the DS procedure to non-adaptive (single observation) measurement at several signal-to-noise ratios $\left(\mathrm{SNR}=\mu^{2}\right)$ with $p=2^{14}$ and $\sqrt{p}=128$ non-zero components. The range of FDP-NDP operating points is surveyed by varying the threshold applied to the non-adaptive measurements and the output of the DS procedure. Recall that largest squared magnitude in a realization of $p$ i.i.d. $\mathcal{N}(0,1)$ variables grows like $2 \log p$, and in our experiment, $2 \log p \approx 20$. Consequently, when the SNR $=20$ we see that both DS and non-adaptive 
measurements are highly successful, as expected. Another SNR level of interest is 8 , since in this case this happens to approximately satisfy the condition $\mu=\sqrt{2 / c_{1}}=\sqrt{2 p / R_{1}}$, which according to the Theorem 2.5 is a critical level for detection using DS. The simulations show that DS remains highly successful at this level while the non-adaptive results are poor. Finally, when the $\mathrm{SNR}=2$, we see that DS still yields useful results. For example, at $\mathrm{FDP}=0.05$, the DS procedure has an average NDP of roughly $80 \%$ (i.e., $20 \%$ of the true components are still detected, on average). This demonstrates the approximate $\log p$ extension of the SNR range provided by DS. Note the gap in the FDP values of the DS results (roughly from 0.75 to 1 ). The gap arises because the the output of DS has a higher SNR and is much less sparse than the original signal, and so arbitrarily large FDP values cannot be achieved by any choice of threshold. Large FDP values are, of course, of little interest in practice. We also remark on the structured patterns observed in cases of high NDP and low FDP (in upper left of figures for SNR $=2$ and SNR $=8$ ). The visually structured 'curves' of NDP-FDP pairs arise when the total number of discoveries is small, and hence the FDP values are restricted to certain rational numbers. For example, if just 3 components are discovered, then the number of false-discoveries can only take the values $0,1 / 3,2 / 3$, and 1 .

Figure 2 compares the average performance of non-adaptive sensing and the DS procedure in terms of the false-discovery rate (FDR) and the non-discovery rate (NDR), which are the average false-discovery and nondiscovery proportions, respectively. For each case, the data are thresholded to achieve a FDR level of 0.05. The plot depicts the NDR as a function of SNR. The ideal NDR is 0, and the plot shows that the DS procedure achieves a much lower NDR over the entire SNR range. The FDRs for both methods are also shown (and both are approximately 0.05 over the entire range). The FDR and NDR were generated by averaging 1000 independent trials at each SNR level, with $p=2^{14}$ and $\sqrt{p}=128$ non-zero components.

5. Concluding Remarks. There has been a tremendous interest in high-dimensional testing and detection problems in recent years. A welldeveloped theory exists for such problems when using a single, non-adaptive observation model $[1,7,11,6]$. However, in practice and theory, multistage adaptive designs have shown promise $[13,17,14,18]$. This paper quantifies the improvements such methods can achieve. We proposed and analyzed a specific multistage design called distilled sensing (DS), and established that DS is capable of detecting and localizing much weaker sparse signals than non-adaptive methods. The main result shows that adaptivity allows 


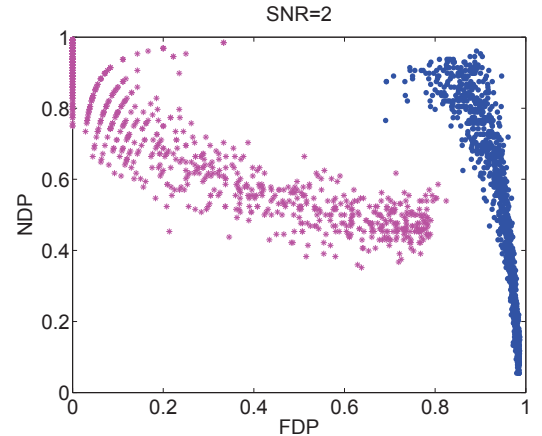

(a)

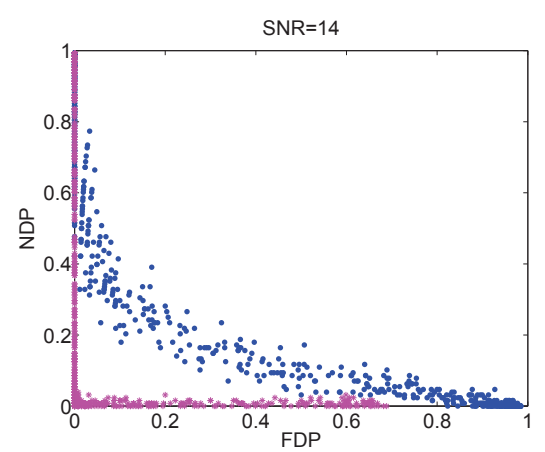

(c)

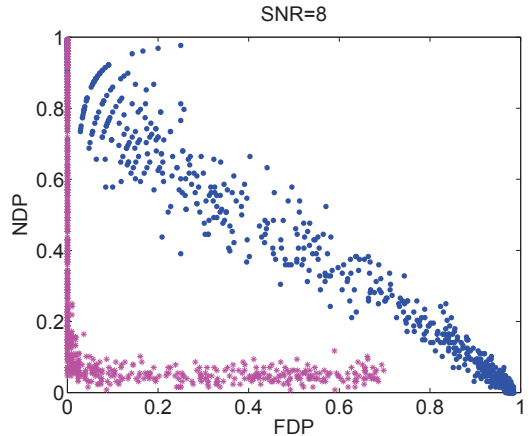

(b)

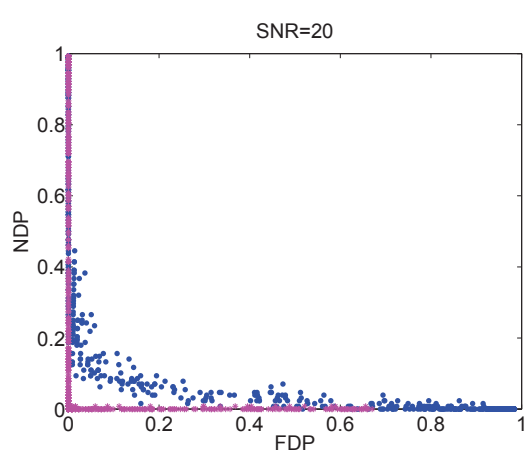

(d)

FIG 1. FDP-NDP performance for $D S$ and non-adaptive sensing. At each SNR, 1000 random sparse signals were randomly generated $(\sqrt{p}$ non-zero component locations selected uniformly at random) and both the non-adaptive observation data and the output of the $D S$ procedure were thresholded at a random positive level. The random threshold level in each trial allows us to explore the full range of FDP and NDP. The FDP and NDP were calculated for both results in each trial. Non-adaptive results are indicated with $\bullet$ and DS results are indicated with $*$.

reliable detection and localization at a signal-to-noise ratio (SNR) that is roughly $\log p$ lower than the minimum required by non-adaptive methods, where $p$ is the problem dimension. To put this in context, suppose one is interested in screening $p=20,000$ genes, then $\log p \approx 10$. Thus, the gains can be quite significant in problem sizes of practical interest, which is why experimentalists often do employ similar methods.

There are several possible extensions to DS. One is to consider even sparser signal models, where the number of nonzero entries is significantly smaller than $p^{1-\beta}$ for $\beta \in(0,1)$, as considered here. In particular, the same asymptotic results stated here follow also for signals whose sparsity levels are as small as a constant times $\log \log \log p$. Indeed, making this choice of 


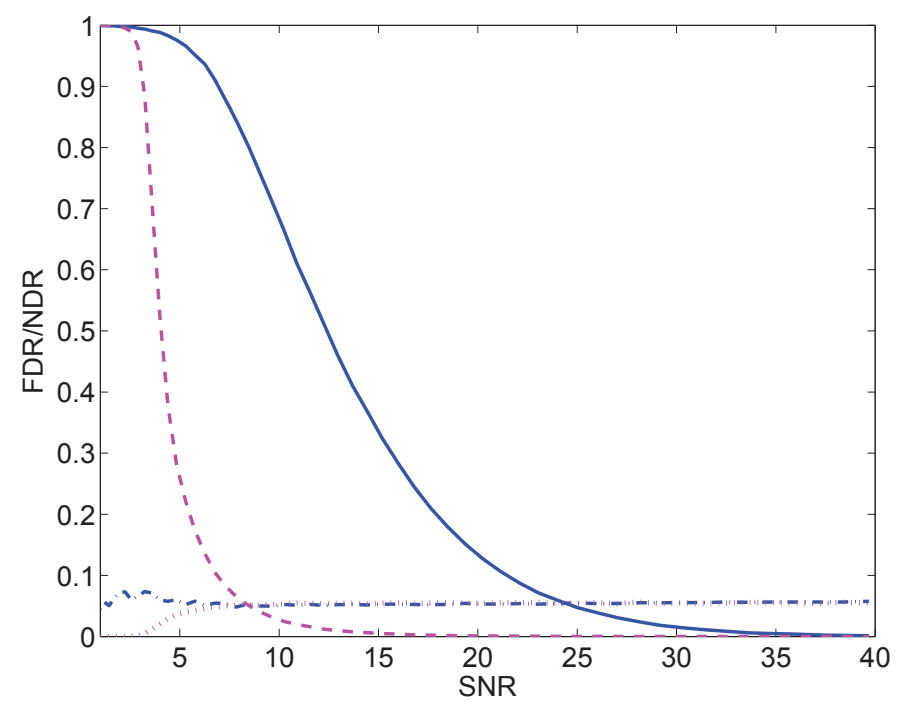

FIG 2. NDR vs. SNR comparison. The non-discovery rate is the average NDP over 1000 independent trials at each $\operatorname{SNR}\left(S N R=\mu^{2}\right)$ and with threshold set to achieve FDR $=0.05$ (FDR is the average FDP). The solid curve depicts the NDR of non-adaptive sensing and the dashed curve depicts the NDR of the DS procedure. At the bottom of the figure, the dash-dot and dot-dot curves show the FDR for non-adapative sensing and DS, respectively (at approximately $5 \%$ for both).

$s_{1}$ in (3.4) leads to the same bound on the $\epsilon_{j}^{2}$ given in (3.5), and this choice is also sufficient to ensure that the probability of the event $\Gamma$ in (3.6) still tends to one. In addition, for this choice of $s_{1}$ the same bound is obtained in (3.7), and the rest of the proof goes through as stated. Another extension is to use DS with alternate measurement models. For example, each measurement could be a linear combination of the entries of $x$, rather than direct measurements of individual components. If the linear combinations are nonadaptive, this leads to a regression model commonly studied in the Lasso and Compressed Sensing literature - see, for example, [15, 2]. However, sequentially tuning the linear combinations leads to an adaptive version of the regression model which can be shown to provide significant improvements, as well [9].

\section{APPENDIX A: THRESHOLDS FOR NON-ADAPTIVE RECOVERY}

In this section we give a proof of Theorem 2.3. We will proceed by considering two cases separately: (i) $r>\beta$ and (ii) $r<\beta$. The analysis of the phase transition point $r=\beta$ is interesting, but it is beyond the scope of this paper. Begin by noticing that in the setting of the theorem the minimax 
optimal support estimation procedure to control the false and non-discovery proportions is a simple coordinate-wise thresholding procedure of the form

$$
\widehat{\mathcal{S}}=\left\{i: y_{i}>\tau\right\}
$$

where $\tau \geq 0$ can be chosen appropriately. A formal proof of this optimality can be done by noting that the class of hypothesis is invariant under permutations (see [11] for details).

Case (i) $r>\beta$ : In this case the signal support can be accurately identified from the observations, in the sense that $\operatorname{FDP}(\widehat{\mathcal{S}})$ and $\operatorname{NDP}(\widehat{\mathcal{S}})$ both converge in probability to zero. For this case we will take $\tau=\tau(p)=\sqrt{2 \alpha \log p}$, where $\beta<\alpha<r$.

Begin by defining $D_{z}$ and $M_{s}$ to be the number of retained non-signal components and the number of missed signal components, respectively. Formally

$$
D_{z}=\sum_{i=1}^{p} \mathbf{1}_{\left\{y_{i}>\tau, x_{i}=0\right\}},
$$

and

$$
M_{s}=\sum_{i=1}^{p} \mathbf{1}_{\left\{y_{i} \leq \tau, x_{i} \neq 0\right\}} .
$$

Note that $D_{z}$ is binomially distributed, that is $D_{z} \sim \operatorname{Bin}\left(p\left(1-p^{-\beta}\right), q_{z}\right)$, where $q_{z}=\operatorname{Pr}\left(y_{i}>\tau\right)$ when $i$ is such that $x_{i}=0$. By noticing that $\tau>0$ and using a standard Gaussian tail bound we have that

$$
q_{z} \leq \frac{1}{\sqrt{2 \pi \tau^{2}}} \exp \left(-\frac{\tau^{2}}{2}\right)=\frac{1}{\sqrt{4 \pi \alpha \log p}} p^{-\alpha}
$$

In a similar fashion note that $M_{s} \sim \operatorname{Bin}\left(p^{1-\beta}, q_{s}\right)$, where $q_{s}=\operatorname{Pr}\left(y_{i} \leq \tau\right)$ when $i$ is such that $x_{i}=\sqrt{2 r \log p}$. Let $Z \sim \mathcal{N}(0,1)$ be an auxiliary random variable. Then

$$
\begin{aligned}
q_{s} & =\operatorname{Pr}(Z+\sqrt{2 r \log p} \leq \tau) \\
& =\operatorname{Pr}(Z \leq \tau-\sqrt{2 r \log p}) \\
& =\operatorname{Pr}(Z>\sqrt{2 \log p}(\sqrt{r}-\sqrt{\alpha}))
\end{aligned}
$$

And so, using the Gaussian tail bound we have

$$
q_{s} \leq \frac{1}{\sqrt{4 \pi \log p}(\sqrt{r}-\sqrt{\alpha})} p^{-(\sqrt{r}-\sqrt{\alpha})^{2}} .
$$


We are ready to show that both $\operatorname{FDP}(\widehat{\mathcal{S}})$ and $\operatorname{NDP}(\widehat{\mathcal{S}})$ converge in probability to zero. Begin by noticing that $\operatorname{NDP}(\widehat{\mathcal{S}})=M_{s} / p^{1-\beta}$. By definition $\operatorname{NDP}(\widehat{\mathcal{S}}) \stackrel{P}{\rightarrow} 0$ means that for all fixed $\epsilon>0$,

$$
\operatorname{Pr}(|\operatorname{NDP}(\widehat{\mathcal{S}})|>\epsilon) \rightarrow 0,
$$

as $p \rightarrow \infty$. Noting that $\operatorname{NDP}(\widehat{\mathcal{S}})$ is non-negative, this can be easily established using Markov's inequality.

$$
\begin{aligned}
\operatorname{Pr}(\operatorname{NDP}(\widehat{\mathcal{S}})>\epsilon) & =\operatorname{Pr}\left(\frac{M_{s}}{p^{1-\beta}} \geq \epsilon\right) \\
& =\operatorname{Pr}\left(M_{s}>\epsilon p^{1-\beta}\right) \\
& \leq \frac{\mathbb{E}\left[M_{s}\right]}{\epsilon p^{1-\beta}} \\
& =\frac{p^{1-\beta} q_{s}}{\epsilon p^{1-\beta}}=\frac{q_{s}}{\epsilon} \rightarrow 0,
\end{aligned}
$$

as $p \rightarrow \infty$ as clearly $q_{s}$ converges to zero (since $r>\alpha$ ). For the false discovery proportion the reasoning is similar. Note that the number of correct discoveries is $p^{1-\beta}-M_{s}$. Taking this into account we have

$$
\operatorname{FDP}(\widehat{\mathcal{S}})=\frac{D_{z}}{p^{1-\beta}-M_{s}+D_{z}} .
$$

Let $\epsilon>0$. Then

$$
\begin{aligned}
\operatorname{Pr} & (\operatorname{FDP}(\widehat{\mathcal{S}})>\epsilon) \\
& =\operatorname{Pr}\left(\frac{D_{z}}{p^{1-\beta}-M_{s}+D_{z}}>\epsilon\right) \\
& =\operatorname{Pr}\left((1-\epsilon) \frac{D_{z}}{p^{1-\beta}}+\epsilon \frac{M_{s}}{p^{1-\beta}}>\epsilon\right) \\
& \leq \frac{\mathbb{E}\left[(1-\epsilon) \frac{D_{z}}{p^{1-\beta}}+\epsilon \frac{M_{s}}{p^{1-\beta}}\right]}{\epsilon} \\
& =\frac{1-\epsilon}{\epsilon} \frac{p\left(1-p^{-\beta}\right) q_{z}}{p^{1-\beta}}+q_{s} \\
& \leq \frac{1-\epsilon}{\epsilon} p^{\beta}\left(1-p^{-\beta}\right) \frac{1}{\sqrt{4 \pi \alpha \log p}} p^{-\alpha}+\frac{1}{\sqrt{4 \pi \log p}(\sqrt{r}-\sqrt{\alpha})} p^{-(\sqrt{r}-\sqrt{\alpha})^{2}},
\end{aligned}
$$

where the last line clearly converges to zero as $p \rightarrow \infty$, since $\beta<\alpha<r$. Therefore we conclude that $\operatorname{FDP}(\widehat{\mathcal{S}})$ converges to zero in probability. 
Case (ii) $r<\beta$ : In this case we will show that no thresholding procedure can simultaneously control the false and non-discovery proportions. Begin by noting that the smaller $\tau$ is, the easier it is to control the non-discovery proportion. In what follows we will identify an upper-bound on $\tau$ necessary for the control of the non-discovery rate. Note that if $\tau=\tau(p)=\sqrt{2 r \log p}$ then $q_{s}=1 / 2$, and therefore

$$
\operatorname{NDP}(\widehat{\mathcal{S}})=\frac{M_{s}}{p^{1-\beta}} \stackrel{a . s .}{\rightarrow} 1 / 2,
$$

as $p \rightarrow \infty$, by the law of large numbers. Therefore a necessary, albeit insufficient, condition for $\operatorname{NDP}(\widehat{\mathcal{S}}) \stackrel{P}{\rightarrow} 0$ is that for all but finitely many $p$

$$
\tau<\sqrt{2 r \log p} .
$$

Similarly, note that the larger $\tau$ is, the easier it is to control the false discovery rate. In the same spirit of the above derivation we will identify a lower-bound for $\tau$ that must necessarily hold in order to control the falsediscovery rate. Recall the previous derivation, where we showed that, for any $\epsilon>0$

$$
\begin{aligned}
\operatorname{Pr}(\operatorname{FDP}(\widehat{\mathcal{S}})>\epsilon) & =\operatorname{Pr}\left((1-\epsilon) \frac{D_{z}}{p^{1-\beta}}+\epsilon \frac{M_{s}}{p^{1-\beta}} \geq \epsilon\right) \\
& \geq \operatorname{Pr}\left((1-\epsilon) \frac{D_{z}}{p^{1-\beta}} \geq \epsilon\right) \\
& =\operatorname{Pr}\left(\frac{D_{z}}{p^{1-\beta}} \geq \frac{\epsilon}{1-\epsilon}\right),
\end{aligned}
$$

where the last inequality follows trivially given that $M_{s} \geq 0$ and, without loss of generality, we assume that $\epsilon<1$. This means that $\operatorname{FDP}(\widehat{\mathcal{S}})$ converges in probability to zero only if $\frac{D_{z}}{p^{1-\beta}}$ also converges in probability to zero. Namely, for any $\epsilon>0$ we must have $\lim _{p \rightarrow \infty} \operatorname{Pr}\left(D_{z} / p^{1-\beta}>\epsilon\right)=0$. In what follows take $\tau=\sqrt{2 r \log p}$. Let $\epsilon>0$ and note that

$$
\begin{aligned}
\operatorname{Pr}\left(\frac{D_{z}}{p^{1-\beta}}>\epsilon\right) & =\operatorname{Pr}\left(D_{z}>\epsilon p^{1-\beta}\right) \\
& =\operatorname{Pr}\left(D_{z}-\mathbb{E}\left[D_{z}\right]>\epsilon p^{1-\beta}-\mathbb{E}\left[D_{z}\right]\right) \\
& =\operatorname{Pr}\left(D_{z}-\mathbb{E}\left[D_{z}\right]>\epsilon p^{1-\beta}-p\left(1-p^{-\beta}\right) q_{z}\right)
\end{aligned}
$$

Define $a=\epsilon p^{1-\beta}-p\left(1-p^{-\beta}\right) q_{z}$. Note that by the Gaussian tail bound, we have

$$
\frac{1}{\sqrt{4 \pi r \log p}}\left(1-\frac{1}{2 r \log p}\right) p^{-r} \leq q_{z} \leq \frac{1}{\sqrt{4 \pi r \log p}} p^{-r},
$$


or equivalently,

$$
q_{z}=\frac{1-o(1)}{\sqrt{4 \pi r \log p}} p^{-r} .
$$

Given this it is straightforward to see that

$$
\begin{aligned}
a & =\epsilon p^{1-\beta}-(1-o(1)) \frac{p\left(1-p^{-\beta}\right)}{\sqrt{4 \pi r \log p}} p^{-r} \\
& =\epsilon p^{1-\beta}-(1-o(1)) \frac{p^{1-r}}{\sqrt{4 \pi r \log p}} \\
& =p^{1-r}\left(\epsilon p^{r-\beta}-\frac{1-o(1)}{\sqrt{4 \pi r \log p}}\right) \\
& =-\frac{p^{1-r}}{\sqrt{4 \pi r \log p}}(1-o(1)),
\end{aligned}
$$

where in the last step we use the assumption that $\beta>r$. Therefore $a \rightarrow-\infty$ as $p$ goes to infinity. Let $p_{0}(\epsilon) \in \mathbb{N}$ be such that $a<0$ for all $p \geq p_{0}(\epsilon)$. Then

$$
\begin{aligned}
\operatorname{Pr}\left(D_{z} / p^{1-\beta}>\epsilon\right) & =\operatorname{Pr}\left(D_{z}-\mathbb{E}\left[D_{z}\right]>a\right) \\
& =1-\operatorname{Pr}\left(D_{z}-\mathbb{E}\left[D_{z}\right] \leq a\right) \\
& \geq 1-\operatorname{Pr}\left(\left|D_{z}-\mathbb{E}\left[D_{z}\right]\right| \geq-a\right) \\
& \geq 1-\frac{\operatorname{Var}\left(D_{z}\right)}{(-a)^{2}}
\end{aligned}
$$

where $\operatorname{Var}\left(D_{z}\right)=p\left(1-p^{-\beta}\right) q_{z}\left(1-q_{z}\right)$ is the variance of $D_{z}$ and the last step uses Chebyshev's inequality. Recalling that $p \geq p_{0}(\epsilon)$ we can examine the last term in the above expression easily.

$$
\begin{aligned}
1-\frac{\operatorname{Var}\left(D_{z}\right)}{(-a)^{2}} & =1-\left(1-q_{z}\right) \frac{p\left(1-p^{-\beta}\right) q_{z}}{a^{2}} \\
& =1-(1-o(1)) \frac{p q_{z}}{a^{2}} \\
& =1-(1-o(1)) \frac{p^{1-r}}{\sqrt{4 \pi r \log p}} \frac{4 \pi r \log p}{p^{2-2 r}} \\
& =1-(1-o(1)) \frac{\sqrt{4 \pi r \log p}}{p^{1-r}} \rightarrow 1
\end{aligned}
$$

as $p \rightarrow \infty$. Therefore we conclude that, for $\tau=\sqrt{2 r \log p}, D_{z} / p^{1-\beta}$ does not converge in probability to zero, and therefore $\operatorname{FDP}(\widehat{\mathcal{S}})$ also does not converge to zero. 
The above result means that a necessary condition for the convergence of $\operatorname{FDP}(\widehat{\mathcal{S}})$ to zero is that for all but finitely many $p$

$$
\tau>\sqrt{2 r \log p} .
$$

This, together with (A.1) shows that there is no thresholding procedure capable of controlling both the false-discovery and non-discovery proportions when $r<\beta$ as we wanted to show, concluding the proof.

\section{APPENDIX B: AUXILIARY MATERIAL}

Lemma B.1. Let $0 \leq f(p) \leq 1 / 2$ and $g(p) \geq 0$ be any sequences in $p$ such that $\lim _{p \rightarrow \infty} f(p) g(p)=0$. Then

$$
\lim _{p \rightarrow \infty}(1+f(p))^{g(p)}=\lim _{p \rightarrow \infty}(1-f(p))^{g(p)}=1 .
$$

Proof. To establish that $\lim _{p \rightarrow \infty}(1+f(p))^{g(p)}=1$ note that

$$
1 \leq(1+f(p))^{g(p)}=\exp (g(p) \log (1+f(p))) \leq \exp (g(p) f(p)),
$$

where the last inequality follows from $\log (1+x) \leq x$ for all $x \geq 0$. As $g(p) f(p) \rightarrow 0$ we conclude that $\lim _{p \rightarrow \infty}(1+f(p))^{g(p)}=1$.

The second part of the result is established in a similar fashion. Note that

$$
\begin{aligned}
\log (1-f(p))=-\log \left(\frac{1}{1-f(p)}\right) & =\log \left(1+\frac{f(p)}{1-f(p)}\right) \\
& \geq-\frac{f(p)}{1-f(p)} \geq-2 f(p)
\end{aligned}
$$

where the last inequality relies on the fact that $f(p) \leq 1 / 2$. Using this fact we have that

$$
1 \geq(1-f(p))^{g(p)}=\exp (g(p) \log (1-f(p))) \geq \exp (-2 f(p) g(p)) .
$$

Taking into account that $g(p) f(p) \rightarrow 0$ establishes the desired result.

Lemma B.2. Let $k=k(p)$ be a positive diverging sequence in $p$, and let $g=g(p)$ be a positive nondecreasing sequence in $p$. For some fixed $a>1$ let $\epsilon_{j}=\epsilon_{j}(p) \leq a^{-j} / g(p)$. If $g(p) \geq a^{-1}$, then

$$
\lim _{p \rightarrow \infty} \prod_{j=1}^{k(p)}\left(1-\epsilon_{j}(p)\right)>0 .
$$


If, in addition, $g(p)$ is any positive diverging sequence in $p$, then

$$
\lim _{p \rightarrow \infty} \prod_{j=1}^{k(p)}\left(1-\epsilon_{j}(p)\right)=1
$$

Proof. Note that

$$
\begin{aligned}
\log \left(\prod_{j=1}^{k(p)}\left(1-\epsilon_{j}(p)\right)\right) & \geq \sum_{j=1}^{k(p)} \log \left(1-\frac{a^{-j}}{g(p)}\right) \\
& =-\sum_{j=1}^{k(p)} \log \left(1+\frac{a^{-j} / g(p)}{1-a^{-j} / g(p)}\right) \\
& \geq-\sum_{j=1}^{k(p)} \frac{a^{-j} / g(p)}{1-a^{-j} / g(p)} \\
& \geq \frac{-1}{1-a^{-1} / g(p)} \sum_{j=1}^{k(p)} a^{-j} / g(p) \\
& =\frac{-1}{g(p)-a^{-1}} \sum_{j=1}^{k(p)} a^{-j}
\end{aligned}
$$

Now, using the formula for the sum of a geometric series, we have

$$
\log \left(\prod_{j=1}^{k(p)}\left(1-\epsilon_{j}(p)\right)\right) \geq \frac{-1}{g(p)-a^{-1}}\left[\frac{a^{-1}\left(1-a^{-k(p)}\right)}{1-a^{-1}}\right],
$$

from which it follows that

$$
\exp \left(\frac{-1}{g(p)-a^{-1}}\left[\frac{a^{-1}\left(1-a^{-k(p)}\right)}{1-a^{-1}}\right]\right) \leq \prod_{j=1}^{k(p)}\left(1-\epsilon_{j}(p)\right) \leq 1 .
$$

Now, assuming only that $g(p)>a^{-1}$, it is easy to see that

$$
\lim _{p \rightarrow \infty} \prod_{j=1}^{k(p)}\left(1-\epsilon_{j}(p)\right)>0,
$$

and if $g(p)$ is any positive diverging sequence in $p$, we have

$$
\lim _{p \rightarrow \infty} \prod_{j=1}^{k(p)}\left(1-\epsilon_{j}(p)\right)=1
$$

as claimed. 


\section{REFERENCES}

[1] F. Abramovich, Y. Benjamini, D. Donoho, and I. Johnstone. Adapting to unknown sparsity by controlling the false discovery rate. Annals of Statistics, 34(2):584-653, 2006.

[2] E. J. Candès. Compressive sampling. In Proc. Int. Congress of Mathematics, volume 3, pages 1433-1452, Madrid, Spain, 2006.

[3] H. Chernoff. A measure of asymptotic efficiency for tests of a hypothesis based on the sum of observations. Annals of Statistics, 23(4):493-507, 1952.

[4] D. Cohn. Neural network exploration using optimal experiment design. In Advances in Neural Information Processing Systems (NIPS), pages 679-686, 1994.

[5] D. Cohn, Z. Ghahramani, and M. Jordan. Active learning with statistical models. Journal of Artificial Intelligence Research, 4:129-145, 1996.

[6] D. Donoho and J. Jin. Higher criticsm for detecting sparse heterogenous mixtures. Annals of Statistics, 32(3):962-994, 2004.

[7] D. Donoho and J. Jin. Asymptotic minimaxity of false discovery rate thresholding for sparse exponential data. Annals of Statistics, 34(6):2980-3018, 2006.

[8] C. Genovese, J. Jin, and L. Wasserman. Revisiting marginal regression. Submitted, 2009.

[9] J. Haupt, R. Baraniuk, R. Castro, and R. Nowak. Compressive distilled sensing: Sparse recovery using adaptivity in compressive measurements. In Proc. 43rd Asilomar Conf. on Signals, Systems, and Computers, Monterey, CA, 2009.

[10] J. Haupt, R. Castro, and R. Nowak. Distilled sensing: selective sampling for sparse signal recovery. In Proc. 12th Conference on Artificial Intelligence and Statistics, Clearwater Beach, FL, 2009.

[11] Y. Ingster. Some problem of hypothesis testing leading to infinitely divisible distributions. Mathematical Methods of Statistics, 6(1):47-69, 1997.

[12] Y. Ingster and I. Suslina. Nonparametric Goodness-of-Fit Testing under Gaussian Models, volume 169 of Lecture Notes in Statistics. Springer, 2003.

[13] H.-H. Muller, R. Pahl, and H. Schafer. Including sampling and phenotyping costs into the optimization of two stage designs for genomewide association studies. Genetic Epidemiology, 31(8):844-852, 2007.

[14] J. Satagopan and R. Elston. Optimal two-stage genotyping in population-based association studies. Genetic Epidemiology, 25(2):149-157, 2003.

[15] R. Tibshirani. Regression shrinkage and selection via the lasso. J. Royal Statist. Soc. B, 58(1):267-288, 1996.

[16] Various. Promising directions in active vision. International Journal of Computer Vision, 11(2):109-126, 1991.

[17] S. Zehetmayer, P. Bauer, and M. Posch. Two-stage designs for experiments with large number of hypotheses. Bioinformatics, 21(19):3771-3777, 2005.

[18] S. Zehetmayer, P. Bauer, and M. Posch. Optimized multi-stage designs controlling the false discovery or the family-wise error rate. Statistics in Medicine, 27(21):4145-4160, 2008. 
J. HAUPT

Electrical and Computer Engineering

Rice University

6100 Main Street

MS-380

Houston, TX 77005

USA

E-MAIL: jdhaupt@rice.edu
R. CAStro

Department of Electrical Engineering

Columbia University

1300 S. W. MUdD

500 W. 120Th ST.

NEW YORK, NY 10027

USA

E-MAIL: rmcastro@ee.columbia.edu

R. NOWAK

EleCtrical ANd Computer EngineERING

UNIVERSITY OF WISCONSIN-MADISON

3627 ENGINEERING HALL

1415 EngineERING Drive

MADISON, WI 53706

USA

E-MAIL: nowak@ece.wisc.edu 